\title{
Quasi-Particle Spectra on Substrate and Embedded Graphene Monolayers
}

\author{
Alexander Feher'1, Eugen Syrkin², Sergey Feodosyev², \\ Igor Gospodarev ${ }^{2}$ and Kirill Kravchenko ${ }^{2}$ \\ ${ }^{1}$ Centre of Low Temperature Physics Faculty of Science P. J. Šafárik University $\mathcal{E}$ \\ Institute of Experimental Physics SAS, \\ ${ }^{2}$ B.I.Verkin Institute for Low Temperature Physics and Engineering NASU, \\ ${ }^{1}$ Slovakia \\ 2Ukraine
}

\section{Introduction}

Graphite, graphene, and compounds based on them are of great interest both as objects of fundamental research and as some of the most promising materials for modern technologies. The two-dimensional form of graphite - graphene - was prepared only very recently, immediately attracting a great deal of attention. Graphene can be deposited on solid substrates and has been shown to exhibit remarkable properties including large thermal conductivity, mechanical robustness and two-dimensional electronic properties. Note that electrons in graphene obey linear dispersion relation resulting in the observation of a number of very peculiar electronic properties. These properties are essentially changed when different defects are introduced into material. Special interest is devoted to graphite intercalated by metals, since in such graphitic systems the temperature of superconducting transition essentially depends on the type of intercalating metal. Besides, the discovery of superconductors as $\mathrm{MgB}_{2}$ and iron pnictides intensified the search for high-temperature superconductivity in materials other than copper oxides. It is known that in the formation of the superconducting state the electron-phonon interaction plays a crucial role (according to the Bardeen-Cooper-Schrieffer theory). Therefore it is necessary to analyze in detail the phonon spectra of pure graphite and to find out how these spectra are influenced by different defects and by intercalation.

This chapter consists of three sections. The first section is devoted to the calculation of the local electronic density of graphene containing a substitutional impurity, vacancy defects due to the substrate surface roughness and adsorbed atoms. The local densities of states for atoms of the sublattice which not contains the vacancy show sharp peaks at energy $\varepsilon=\varepsilon_{F}$ ( $\varepsilon_{F}$ is the energy of the Dirac singularity for ideal graphene). Local spectral densities of atoms of the sublattice which contains the vacancy conserve the same Dirac singularity as is observed in an ideal graphene.

The second section will present our model, which allows to quantitatively describe the phonon spectrum of graphite and to determine the relaxation of force constants for the formation of the surface of the sample and the formation of thin films (bigraphene, 
trigraphene, etc.). Bending stiffness is calculated at the microscopic level for graphene monolayers, the characteristic features of the phonon density of states are identified and atomic displacements along the graphene monolayers and perpendicularly to them are determined. The mean square amplitude of atomic vibrations will be calculated, allowing us to make conclusions on the stability of the bigraphene lattice up to room temperatures. Note that the phonon spectra of graphene monolayers deposited on the substrate are determined by the substrate and they bear very little information on the vibrational characteristics of the carbon atoms.

Finally, the third section will deal with the changes introduced into phonon spectrum of graphite intercalated by different metals. In graphite the spectral density of phonons polarized along the c-axes shows a $V$-type singularity analogous to the so-called Dirac singularity typical for the graphene electron density states. We study the appearance of the quasilocalized states which increase the number of phonons near this singularity. Our investigations make it possible to predict the general properties of phonon and electron spectra for graphite intercalated with different metals. In addition, analyzing the changes in phonon spectra of graphite intercalated with various metals, using the BCS theory, we have proposed, taking into account dynamic properties of these layered systems, a possible way leading to the increase of $T_{c}$.

\section{Electronic spectra of graphene with defects}

It is well known (Kossevich, 1999) that in flat monoatomic layers the mean-square amplitudes of the atoms in the normal direction to the layer plane diverge even at $T=0$. Therefore, graphene monolayers can not exist as a flat formation in the free state. From the substrate only small scales can break off, which immediately become crimped (Meyer et al., 2007). So we can study and practically apply only such a graphene, which is deposited on a certain substrate, which provides for the stability of the plane graphene monolayers (Novoselov et al., 2005; Castro et al., 2009; Peres et al., 2007). In the study of the electronic properties of graphene a dielectric (often silicon) substrate is used, since it does not change its electronic spectrum, but greatly increases the possibility of introducing different kind of defects into it. For example, in graphene spray-deposited on silicon, the vacancies can appear (Shangduan et al., 2008), whereas in graphite (a set of weakly interacting graphene monolayers) and carbon nanotubes vacancies are "healed", forming a stacking fault with local fivefold symmetry axis (Chen et al., 2007).

This section presents the results of the calculations of local electron densities of atoms of graphene containing a substitutional impurity, vacancy defects due to the irregularities of the substrate and adsorbed atoms.

According to its electronic properties graphene is a two-dimensional semiconductor with zero gap. The fact that the charge carriers in graphene are formally described by the Dirac equation rather than the Schrödinger equation is caused by the symmetry of the crystal lattice of graphene, which consists of two equivalent carbon sublattices $A$ and $B$ (left part of Fig. 1). Electronic subbands formed by the combination of symmetric and antisymmetric wave functions for the two sublattices intersect at the edge of the Brillouin zone, which leads to a cone-shaped energy spectrum near the Dirac points $K$ and $K^{\prime}$ (right part of Fig. 1), so that the dynamics of electrons is described by the linear dependence of energy on the quasimomentum (in ordinary metals and semiconductors the dispersion dependence is parabolic). 


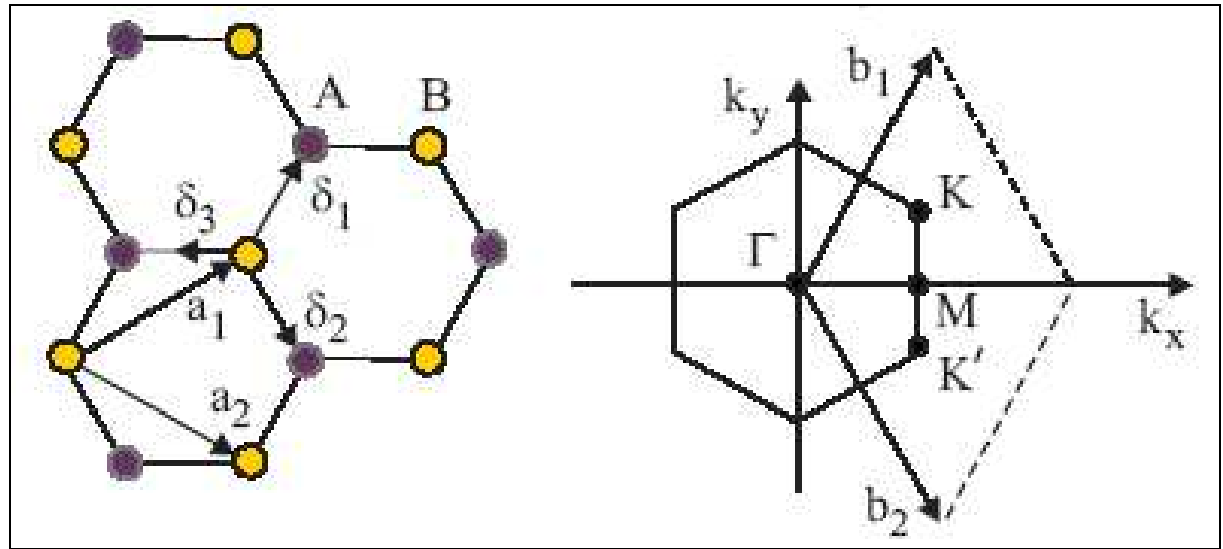

Fig. 1. Structure of the crystal lattice of graphene (left) and its first Brillouin zone (right)

The electronic spectrum of graphene can be described in the strong coupling approximation, taking into account only the interaction of nearest neighbours (Castro et al., 2009; Peres et al., 2007; Skrypnyk \& Loktev, 2006; Skrypnyk \& Loktev, 2008; Bena \& Kivelson, 2005). The corresponding Hamiltonian has the form

$$
H=\sum_{i} \varepsilon_{i}|i\rangle\left\langle i\left|-\sum_{i, j} J_{i j}\right| i\right\rangle\langle j|,
$$

where the indices $i$ and $j$ denote the sites of a two-dimensional lattice, $\varepsilon_{i}$ is the energy of a particle at the $i$ site, and $J_{i j}$ is the so-called overlap integral. Fig. 2a presents the density of electronic states of graphene (curve 1, red line) and the real part of the Green's function (curve 2, blue line). These calculations were made using the method of Jacobi matrices (Peresada et al., 1975). Note that in a perfect graphene, due to the physical equivalence of the atoms of both sublattices, the local Green's function $G(\varepsilon, i)=\left\langle i\left|(\varepsilon \hat{I}-\hat{H})^{-1}\right| i\right\rangle$ coincides with the full function $G(\varepsilon)=\lim _{N \rightarrow \infty} \frac{1}{N} \sum_{i=1}^{N}\left\langle i\left|(\varepsilon \hat{I}-\hat{H})^{-1}\right| i\right\rangle$.

A feature on the density of states for $\varepsilon=\varepsilon(K)$ (namely, that the value $\varepsilon(K)$ corresponds to $\varepsilon_{F}$ which is the Fermi energy in graphene) determines the behavior of the real part of the Green's function in the vicinity of $\varepsilon_{F}$. For a wide class of perturbations caused by defects we can find quasilocalized states, using the Lifshits equation in the interval $[-\varepsilon(M), \varepsilon(M)]$ (in this model $\varepsilon(M)=J$ ) (Lifshits, 1945). This equation, which determines their energy, can be written as (Kossevich, 1999; Peresada et al., 1975)

$$
\operatorname{Re} G(\varepsilon)=S\left(\varepsilon, \Lambda_{i k}\right)
$$

where the $S\left(\varepsilon, \Lambda_{i k}\right)$ function is determined by the operator of the perturbation $\hat{\Lambda}\left(\Lambda_{i k}\right.$ are matrix elements of this operator on defined basis). 


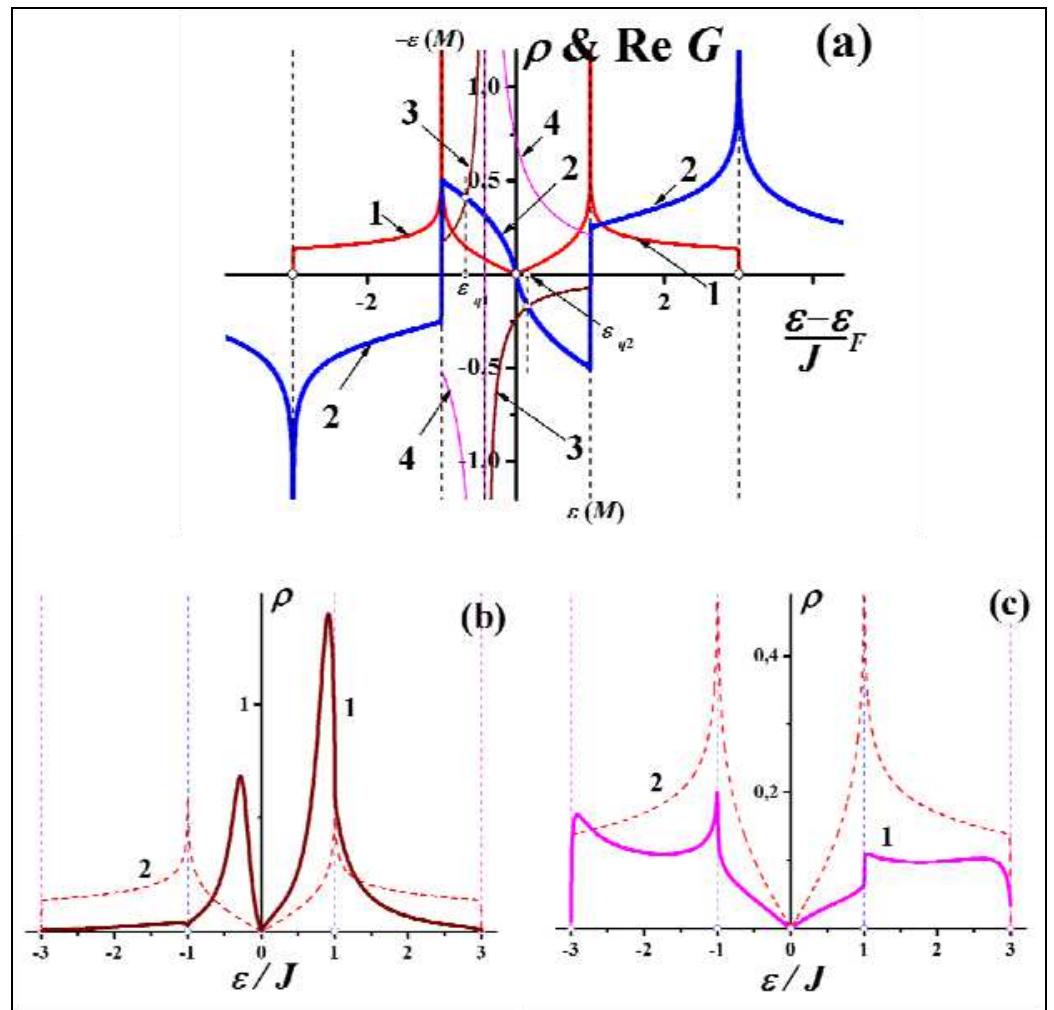

Fig. 2. (a) Density of states (curve 1) and real part of the Green's function (curve 2) in ideal graphene, curves 3 and 4 represent functions $S(\varepsilon)$ for substitutional impurities of nitrogen and boron; (b) and (c) are spectral densities of nitrogen and boron impurities, respectively (curves 1), curves 2 in these figures are the densities of states for ideal graphene

Fig. 2a illustrates the graphic solutions of the Lifshits equation for graphene containing isolated substitutional impurities, namely the atoms of nitrogen and boron. Local spectral densities $\rho(\varepsilon, i) \equiv \frac{1}{\pi} \lim _{\gamma \downarrow 0} \operatorname{Im} G(\varepsilon+\mathrm{i} \gamma, i)$ of impurity atoms are calculated in (Peres et al., 2007). For an isolated substitutional impurity, different from the host lattice atom, the energy of the impurity site $i=0\left(\varepsilon_{0}=\tilde{\varepsilon}\right)$ and the overlap integral $J_{i 0}=(1+\eta) J$, the function $S(\varepsilon, \tilde{\varepsilon}, \eta)$ has the form

$$
S(\varepsilon, \tilde{\varepsilon}, \eta)=\frac{(1+\eta)^{2}}{\tilde{\varepsilon}+\varepsilon \eta(2+\eta)} .
$$

For nitrogen impurity (according to (Peres et al., 2007) in such a case $\tilde{\varepsilon}-\varepsilon(K) \approx-0.525 \mathrm{~J}$, and $\eta \approx-0.5$ (the dependence $S(\varepsilon)$ is shown in Fig. 2a, curve 3). For equation (2), as seen from the figure, the solution for interval $[-\varepsilon(M), \varepsilon(K)]$ is point $\varepsilon_{q 1}$ and for interval 
$[\varepsilon(K), \varepsilon(M)]$ it is the point $\varepsilon_{q 2}$. Local densities of states $\rho(\varepsilon, i)$ of nitrogen impurities calculated in (Peres et al., 2007) have quasi-local maxima in both of these intervals (Fig. 2b).

Although, because of differences in these intervals, the imaginary part of the Green's function is different from zero, the location of quasi-local maxima are different from $\varepsilon_{q 1}$ and $\varepsilon_{q 2}$, and the presence or absence of solutions of the Lifshits equation in the interval under the given parameters of the defect determines the presence or absence of quasilocalized states in this interval.

So, as discussed in (Peres et al., 2007), for the impurity boron $(\tilde{\varepsilon}-\varepsilon(K) \approx 0.525 \mathrm{~J} ; \eta \approx 0.5)$ the quasilocalized states are absent in the interval $[-\varepsilon(M), \varepsilon(M)]$, as seen in Fig. 2c. In this case equation (2) also has no solutions in the interval $[-\varepsilon(M), \varepsilon(M)]$ (corresponding dependence $S(\varepsilon)$ is shown in Fig. 2a as curve 4). Local Green's function of the boron impurity has a peculiarity outside the band of quasi-continuous spectrum, corresponding to a so-called local level in the energy spectrum. Therefore, the area under the curve 2 is smaller than the area under the curve 1, the difference being the residue at local level, what is clearly seen in Fig. 2c.

The function $\operatorname{Re} G(\varepsilon)$ allows us to conclude that the solution of equation (2) in the interval $[-\varepsilon(M), \varepsilon(M)]$ exists for a wide class of perturbations. Fig. 3 shows the local density of states of an atom adsorbed on a graphene monolayer for different values $\tilde{\varepsilon}$ and $\eta$, characterizing the interaction of this atom with carbon atoms. It is clear that the weakening of the adsorbed atom interaction with its nearest neighbors causes that on the local density of states sharp resonance peaks are formed near the $\varepsilon=\varepsilon_{F}$.

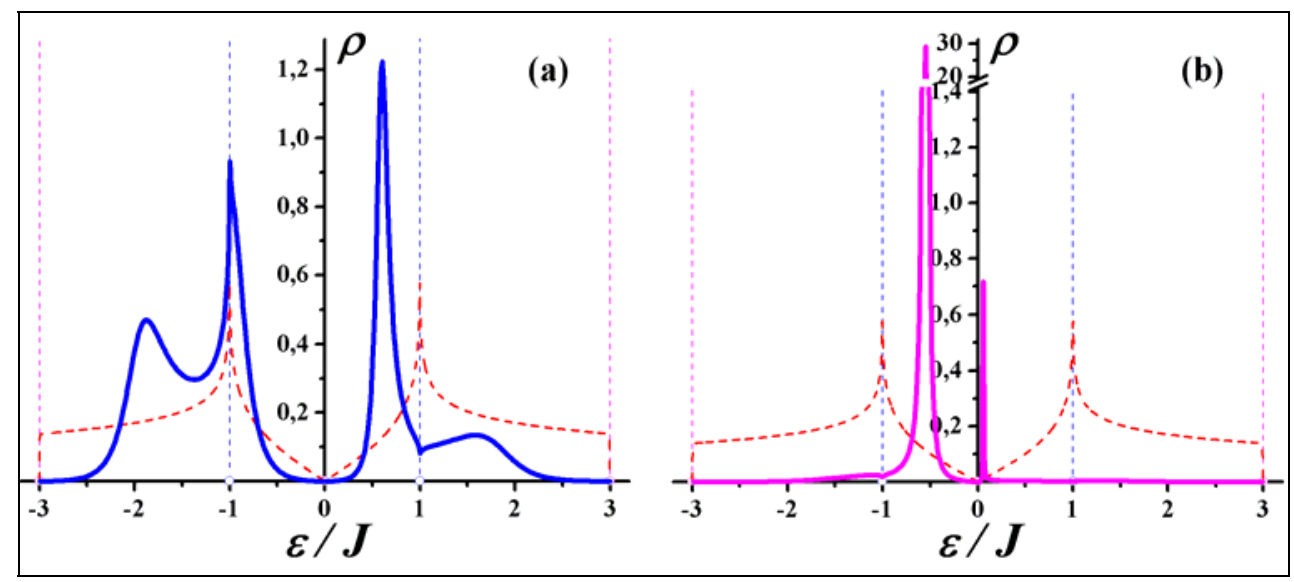

Fig. 3. Local density of states of atoms adsorbed on graphene film: (a) $\tilde{\varepsilon}=-0.5 \mathrm{~J}, \quad \eta=-0.5$; (b) $\tilde{\varepsilon}=-0.5 \mathrm{~J}, \quad \eta=-0.9$; dashed lines represent the density of states of ideal graphene

Thus, the behaviour of the real part of the Green's function in graphene for $-\varepsilon(M) \leq \varepsilon \leq \varepsilon(M)$ shows high sensitivity of the density of states at given energies to various perturbations caused by defects and other changes in the crystal structure, in particular, the possible formation of localized excitations with energies close to the Fermi energy. Generally speaking, it is not important whether this perturbation is degenerate 
(function $S\left(\varepsilon, \Lambda_{i k}\right)$ can be written explicitly) or nondegenerate: then quasilocalized states will arise enriching the electronic spectrum near $\varepsilon=\varepsilon_{F}$. As an example of the influence of such nondegenerate perturbation we consider the local spectral density of atoms of different coordination spheres at an isolated vacancy in the graphene monolayer.

The calculation results are partially presented in Fig. 4, showing the local density $\rho(\varepsilon, i)$ of states for the nearest, second, seventh and tenth neighbours of vacancies (Feher et al., 2009).
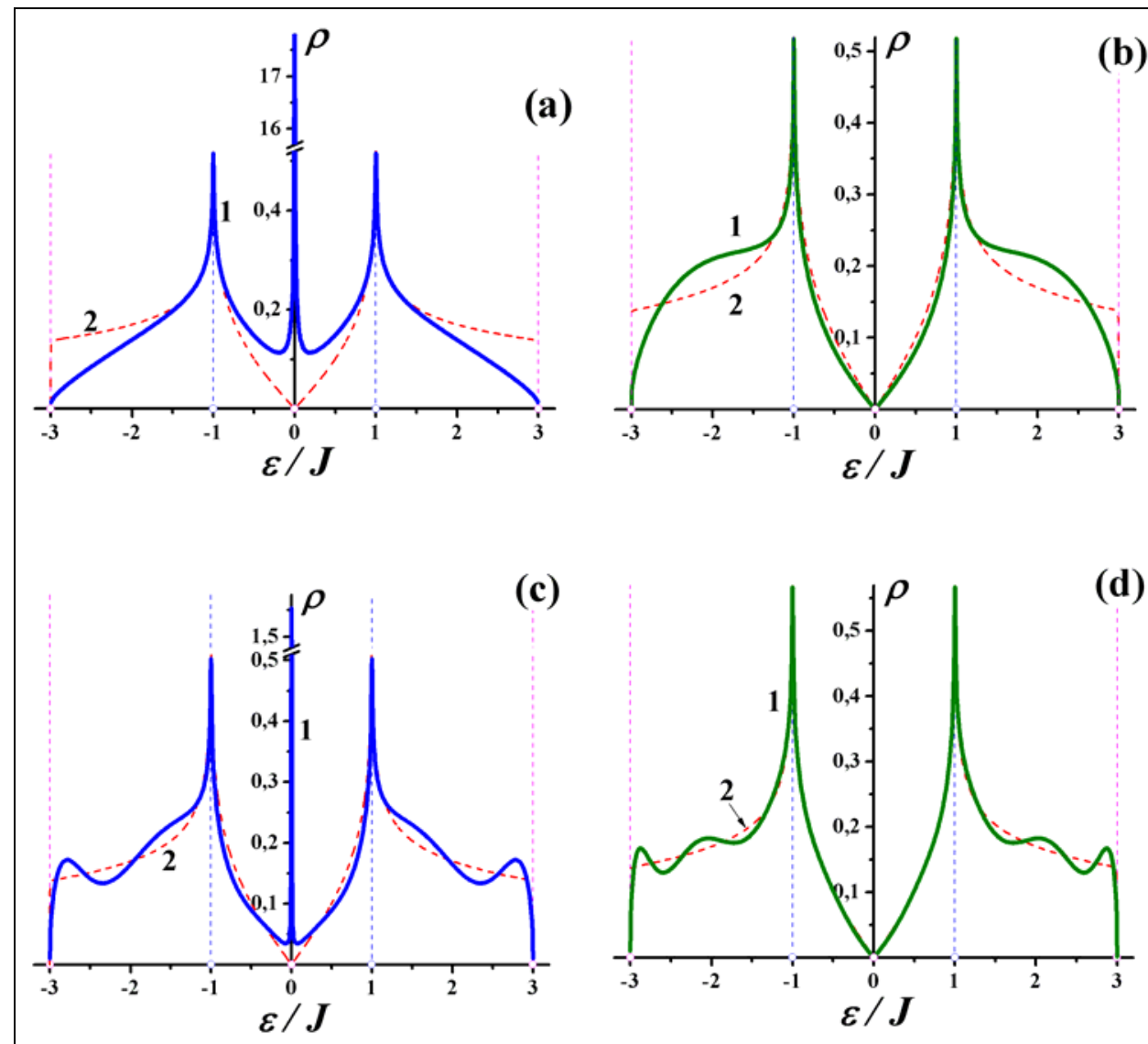

Fig. 4. Local density of electronic states of neighboring vacancies (curves 1); (a) the nearest, (b) the second, (c) the seventh, (d) the tenth; curve 2 (dashed lines) represent the density of electronic states of perfect graphene

Odd neighbours of a vacancy belong to the sublattice which does not contain vacancies (let this be the sublattice $B$ in Fig.1). In the local densities of atoms of this sublattice $\rho(\varepsilon, B)$ a sharp peak occurs at $\varepsilon=\varepsilon(K)=\varepsilon_{F}$. Local density of states in the sublattice A retains, for $\varepsilon=\varepsilon(K)=\varepsilon_{F}$, the same Dirac singularity as in the perfect graphene. This was proved in (Feher et al., 2009) by using the formula obtained in (Kotlyar \& Feodosyev, 2006), which relates an arbitrary matrix element of the Green's function $G(\varepsilon, i)$ in the formalism of the 
method of Jacobi matrices to the first diagonal element of this function, i.e. to the local Green's function $\rho(\varepsilon, A)$. Interaction with second neighbours would change the local density of states $\rho(\varepsilon, A)$ near $\varepsilon=\varepsilon_{F}$, but the magnitude of this change is in the order of the interaction, which is small.

The surface roughness of the substrate may cause the anisotropy of the interatomic interaction, since the overlap integrals $J_{i k}$ will be different for the interaction of the same atom with its various neighbours. Note that in models of "anisotropic graphene" (when the anisotropy of overlap integrals is retained over long-range order) a gap between the bands of the electronic spectrum as well as the formation of additional logarithmic singularities due to the displacement of the intersection of electronic branches inside the first Brillouin zone may appear. Here we consider the case when the atom with the anisotropy of the overlap integrals is an isolated defect.

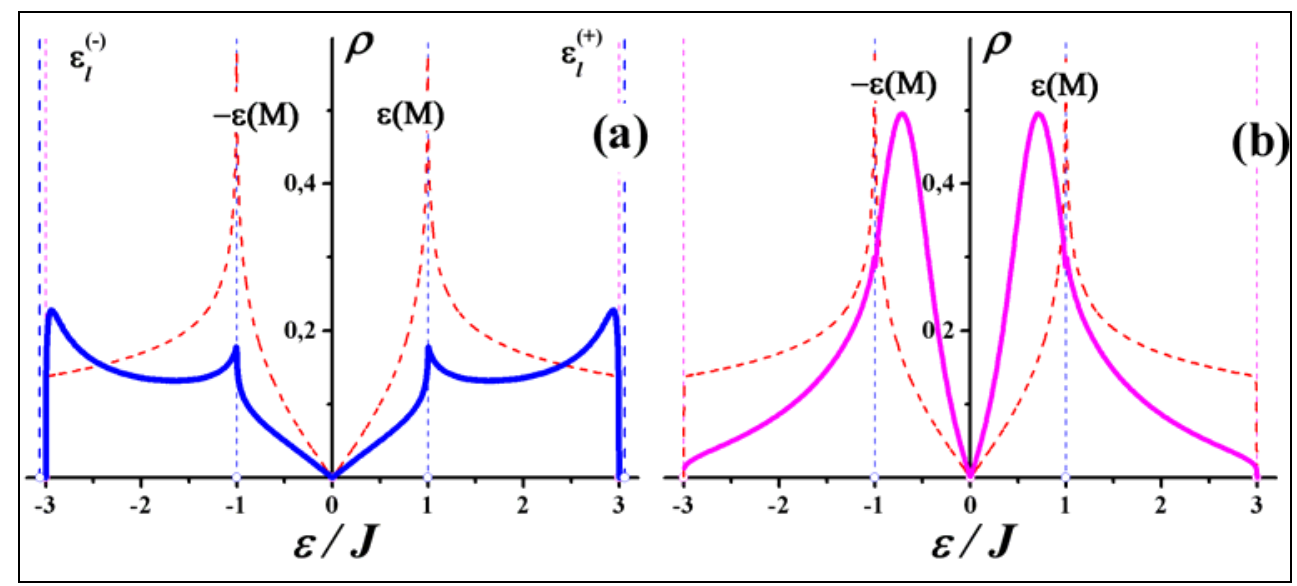

Fig. 5. Local density of states of an isolated defect in lattice of carbon atoms with an anisotropic interaction between nearest neighbours: (a) $\eta=0.5$; (b) $\eta=-0.5$; dashed lines are density of states of a perfect isotropic graphene

Let the interaction of the atom with one of the nearest neighborus be described by the overlap integrals $J$ and $\tilde{J}=(1+\eta) J$. Fig. 5 shows the corresponding local densities of states of the atom. Fig. 5a shows the density of states for $\eta=0.5$, corresponding to the enhanced that is, the interaction increased. In this case the electronic spectrum contains, along with the quasi-continuous part, also two symmetric discrete levels $\varepsilon_{l}^{( \pm)} \approx \pm 3.0698 \mathrm{~J}$ (quasi-continuous band of spectrum in this case is $-3 J \leq \varepsilon \leq 3 J$ ). The values of $\varepsilon_{l}^{( \pm)}$denote the local levels of spectra. Residues at these points (so-called intensities of discrete levels) are $\mu_{l}^{( \pm)} \approx 0.139$. The area under the curve $\rho(\varepsilon)$ is in this case less than unity, the rest being in the sum of the intensities of discrete levels. For $\eta=-0.5$ the electronic states of an anisotropic defect lie in the band of a quasi-continuous spectrum and the interval $-\varepsilon(M) \leq \varepsilon \leq \varepsilon(M)$ contains essentially more electronic states as compared to the perfect isotropic graphene. Thus, controlling the roughness of the substrate could be a promising method for tuning the electronic spectrum of graphene. 


\section{Phonon spectra and vibrational characteristics of carbon nanolayers}

The phonon spectra of graphene monolayers deposited on the substrate are determined by the substrate and have very little value for the determination of the vibrational characteristics of carbon atoms. At the same time, carbon nanofilms, consisting of several graphene monolayers (starting with two, i.e. bigraphene) are stable up to room temperature. This section describes the phonon spectra and vibrational characteristics of bulk samples of graphite and their transformation for decreasing number of layers, down to carbon nanofilms. It outlines our model which allows to quantitatively describe the phonon spectrum of graphite and to determine the relaxation of the force constants in the formation of the sample surface and the formation of ultrathin films (bigraphene, trigraphene, etc.). Flexural stiffness was calculated at the microscopic level for graphene monolayers, as well as the characteristic features of the phonon density of states and the contributions to the atomic displacements along the graphene monolayers and in the perpendicular direction to them were identified. We also calculated the mean square amplitudes of atomic vibrations, explaining the reason of the stability of the lattice bigraphene up to the room temperature.

It is known that the crystal of graphite is a strongly anisotropic layered crystal. Strong anisotropy is, on the one hand, due to a significant difference in the interatomic distances between nearest neighbours in the layer plane and in adjacent layers, and, on the other hand, due to different types of force bonds (covalent, metallic and van der Waals bonds).

Graphite consists of graphene monolayers, the atoms of which form a regular hexagon. The atoms of one of the sublattices are located in the centers of the triangles of the other sublattice. Bravais vectors, lying in the basal plane, can be selected as follows: $R_{1}=a_{0}\left(\frac{\sqrt{3}}{2} ; \frac{1}{2} ; 0\right)$ and $R_{2}=a_{0}\left(\frac{\sqrt{3}}{2} ;-\frac{1}{2} ; 0\right)$, where $a_{0} \approx 2.45 \AA$. The period of the graphite lattice along the axis $c$, in the direction perpendicular to the graphene layers, is equal to twice the interlayer distance, i.e. $R_{3}=c_{0}(0 ; 0 ; 1)$, the parameter $c_{0} \approx 6.7 \AA$ (Fig. 6). The atoms of different sublattices of the graphite basal plane are differently situated with respect to the atoms of neighboring planes, leading to the differences in the interaction between atoms belonging to different sublattices and to their physical inequivalence. Thus, there will be different local Green's functions corresponding to these atoms and determining their vibrational characteristics, such as the mean-square displacements of atoms along different crystallographic directions.

Strong anisotropy of interatomic interaction causes a number of typical distinguishing features in the behaviour of the phonon spectra and vibrational characteristics (Kosevich et al., 1994). For example, the elastic moduli $\mathrm{C}_{33}$ and $\mathrm{C}_{44}$ associated with displacements along the $c$ axis and determining the speed of sound propagating or polarized along this direction, are from about 30 for about 300 times smaller than the elastic moduli $\mathrm{C}_{11}$ and $\mathrm{C}_{66}$, determining the speed of sound propagating and polarized in the basal planes (Nicklow et al., 1972; Belen'kii, et al., 1988). Therefore, if the propagation of the vibrations polarized along $\boldsymbol{c}$ in the basal $\boldsymbol{a} \boldsymbol{b}$ plane would have the nature of a sound wave and not a quasi-flexural wave, the mean-square displacements of atoms in a given direction would attain, even at low temperatures, the values corresponding to the melting of the crystal. That is, the mere existence of solid graphite at room temperature indicates that the fluctuations are essentially determined by the restoring forces acting on the atom from other atoms, lying in the same layer. These restoring forces are due to the noncentral interatomic interaction and indicate the presence of elastic stresses in the graphene layers that form the crystal lattice of graphite. 


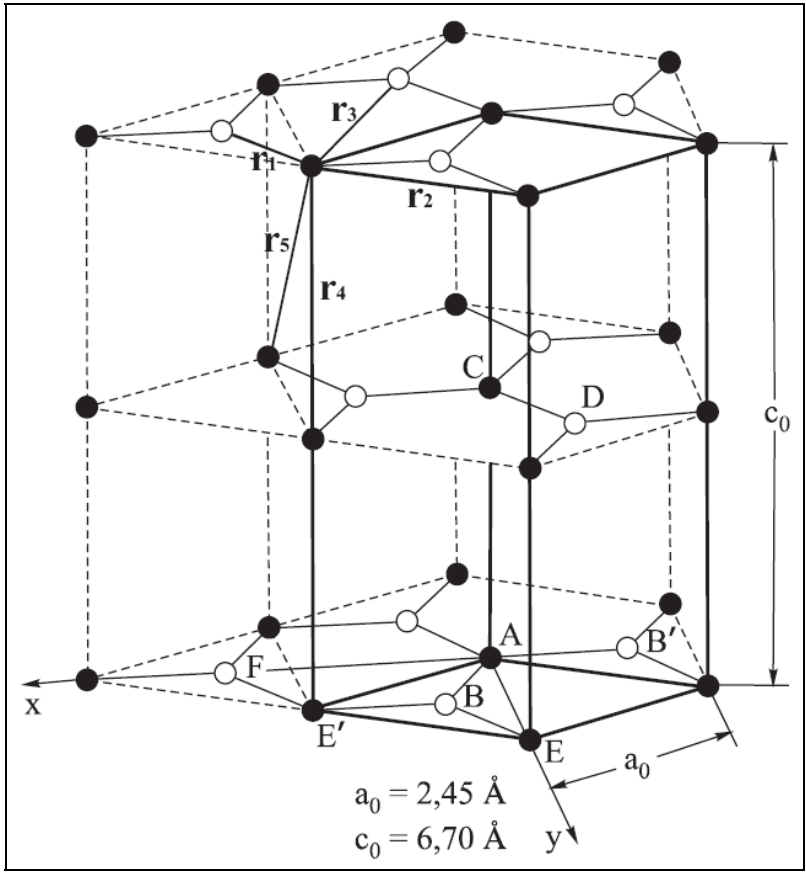

Fig. 6. The structure of the crystal lattice of graphite

In the long-wave region the dispersion law of the transverse phonon mode (TA) of a strongly anisotropic layered crystal, whose frequency vanishes at $k=0$ and which corresponds to the waves propagating in the $a b$ plane and polarized along $c$ (i.e. quasiflexural mode) has the form

$$
\omega_{T_{3}}^{2}(\vec{k})=\frac{C_{44}}{\rho} k^{2}+\frac{\kappa}{m} k^{4} .
$$

Here $\rho$ is the density of graphite, $m$ is the mass of the carbon atom and the coefficient $\kappa$ characterizes the bending stiffness of layers. The deviation described by the second term of (4), characterising quasi-flexural wavelength of the (TA) dispersion curve is clearly seen on dispersion curves obtained from inelastic neutron scattering data (Kosevich et al., 1994). The fact that we can examine each layer of graphite as plane means that in the crystal the condition

$$
\sigma_{i z} n_{i z}=0
$$

is fulfilled, where the coordinate $\mathrm{z}$ is chosen along the $c$ axis and the coordinates $\mathrm{x}$ and $\mathrm{y}$ in the basal plane.

Bending stiffness of flat graphene monolayers, which provides a characteristic distortion of the dispersion curve (Kosevich et al., 1994), does not depend on the interlayer interaction and can be determined from the consideration of only one isolated graphene monolayer. When the condition of equilibrium of a flat layer, which has for an isolated graphene 
monolayer the form $\beta_{1 z}+6 \beta_{2}+4 \beta_{3}=0$, is fulfilled, the bending stiffness $\kappa$ can be written as (Syrkin et al., 2009)

$$
\kappa=\left(\frac{a_{0}}{8}\right)^{2} \sqrt{\frac{6 \beta_{3}}{m} \frac{\beta_{1 z}+6 \beta_{3}}{\beta_{1 z}+\beta_{3}}} .
$$

In (Syrkin et al., 2009) the expressions for the flexural rigidity in some other layered structures are also given. For the layered hexagonal close-packed crystal

$$
\kappa=a^{2} \sqrt{\frac{3}{2}\left(\beta_{1 z}+2 \beta_{2 z}\right)} .
$$

In the layered tetragonal lattice the flexural rigidity is anisotropic (depending on the direction of propagation of flexural waves in the layer plane)

$$
\kappa=\left(\frac{a}{2}\right)^{2} \sqrt{-\left(\beta_{1 z}+2 \beta_{2 z}\right)-2\left(\beta_{1 z}+4 \beta_{2 z}\right) \sin ^{2} 2 \phi},
$$

where $\phi$ is the polar angle in the basal plane, measured from the $x$-axis in the counterclockwise direction.

Therefore, when describing the intralayer interaction in the graphite, the central and noncentral interactions between atom and its first, second and third neighbours must be considered. Accounting for more distant neighbours in the basal plane has no meaning as to the accuracy of the adiabatic approximation. In describing the weak interlayer interaction it is natural to limit of only to the interaction between atoms from neighbouring layers, being at distances $r_{4}$ or $r_{5}$, where the value $r_{5}$ is only slightly larger than $r_{4}$ (see Fig. 6). For the nearest neighbours in the basal plane $\left(\Delta=r_{1}\right)$, whose interaction is determined by the superposition of covalent and metallic bonds, the force matrix is characterized by all three parameters of interaction. The bond between more distant neighbours $\left(\Delta=r_{2}, r_{3}, r_{4}, r_{5}\right)$ can be regarded as a van der Waals one and described by an isotropic pair potential.

The proposed model involves eleven force constants. These constants can be found using follows data: four moduli of elasticity, the condition of symmetry of these moduli under the permutation of pairs of indices, the neutron diffraction data (Kosevich et al., 1994), the inelastic X-ray scattering data (Maultzsch et al., 2004) and the Raman scattering data (Dresselhaus et al., 2002). The values of force constants (see Table 1) that characterize the interaction between the atoms of graphite can be thus unambiguously obtained and checked.

\begin{tabular}{|c|c|c|c|c|c|}
\hline \multirow{2}{*}{} & \multicolumn{5}{|c|}{$\Delta$} \\
\cline { 2 - 6 } & $r_{1}$ & $r_{2}$ & $r_{3}$ & $r_{4}$ & $r_{5}$ \\
\hline$\alpha .10^{3} \mathrm{~N} / \mathrm{m}$ & 337.882 & 50.476 & 19.647 & 2,581 & 0.371 \\
\hline \multirow{2}{*}{$\beta .10^{3} \mathrm{~N} / \mathrm{m}$} & $\beta_{x}=170.864$ & \multirow{2}{*}{10.149} & 8.661 & 0.0654 & 0.0353 \\
\cline { 2 - 4 } & $\beta_{y}=96.375$ & & & & \multirow{2}{*}{0.063} \\
\hline
\end{tabular}

Table 1. The force constants of graphite 
Interlayer van der Waals interaction can be described by the Lennard-Jones potential, which allows, together with the help of expressions for $C_{33}$ and $C_{44}$, to find constants responsible for the interaction between the nearest atoms of neighbouring layers. Moreover, from the values of force constants the parameters of this potential can be determined, which is important for the calculations of the surface relaxation in graphite $(\sigma \approx 3.092 \AA, \varepsilon \approx 152.3 \mathrm{~K})$. It is obvious that the flat form of a free graphene monolayer is not stable. Therefore, we analysed the phonon spectrum and the rms (root mean squares) amplitudes of displacements in the films consisting of two and three graphene monolayers. Results of the experimental studies of graphene bilayer films are given in (Morozov \& Firsov, 2009).

It was shown that in the graphite the interlayer interaction contains both central and noncentral forces, therefore the formation of the surface can not be described by the LifshitzRosenzweig model (as a solution of the stochastic boundary problem) and will be characterized by the reconstruction and relaxation processes. At the same time, it is natural to assume that the breakage of weak interlayer van der Waals bonds will change neither the distance between atoms in graphene layers nor the force constants that characterize the intralayer interaction.

In fact, the surface reconstruction and relaxation will lead to changes in the interlayer distances and force constants $\alpha_{4}, \beta_{4}, \alpha_{5}$ and $\beta_{5}$ characterizing the interlayer interaction.

Condition (5) leads to the same ratio between the force constants and lattice parameters as the condition $C_{13}=C_{31}$. For thin films with $N$ monolayers this condition takes the form (Gospodarev et al., 2010)

$$
\beta_{1}+6 \beta_{2}+4 \beta_{3}=\frac{N}{N-1} \frac{2}{3 a^{2}}\left[r_{4}^{2} \beta_{4}-9\left(r_{1}^{2}-2 r_{4}^{2}\right) \beta_{5}\right] .
$$

From the parameters of the Lennard-Jones potential for the considered graphite thin films the interlayer distance and the force constants describing the interlayer interaction can be easily found:

for two-layer film (bigraphene):

$$
\begin{aligned}
& r_{4} \approx 3.636 \AA ; \mathrm{a}_{4} \approx 0.373 \mathrm{~N} / \mathrm{m} ; \beta_{4} \approx 0.0035 \mathrm{~N} / \mathrm{m} ; \\
& r_{5} \approx 3.902 \AA ; \mathrm{a}_{4} \approx-0.009 \mathrm{~N} / \mathrm{m} ; \beta_{4} \approx 0.004 \mathrm{~N} / \mathrm{m} ;
\end{aligned}
$$

for three-layer film (trigraphene):

$$
\begin{gathered}
r_{4} \approx 3.453 \AA ; \alpha_{4} \approx 1.585 \mathrm{~N} / \mathrm{m} ; \beta_{4} \approx-0.0015 \mathrm{~N} / \mathrm{m} ; \\
r_{5} \approx 3.713 \AA ; \alpha_{4} \approx 0.016 \mathrm{~N} / \mathrm{m} ; \beta_{4} \approx 0.004 \mathrm{~N} / \mathrm{m} .
\end{gathered}
$$

Fig. 7 presents the phonon densities of states of bigraphene (Fig. 7a) as well as the contributions to them from the atomic displacements along the layers (Fig. 7b) and perpendicular to them (Fig. 7c).

In each figure the dashed line shows the corresponding characteristics of an infinite graphite sample (Gospodarev et al., 2009). Densities of states of the film and bulk samples are practically the same, significant differences were observed only in the frequency range in which the phonon spectrum of graphite resembles that of a three-dimensional system and the interaction between the vibrational modes polarized in the plane of the layers and perpendicularly to the layers is sufficiently large. 


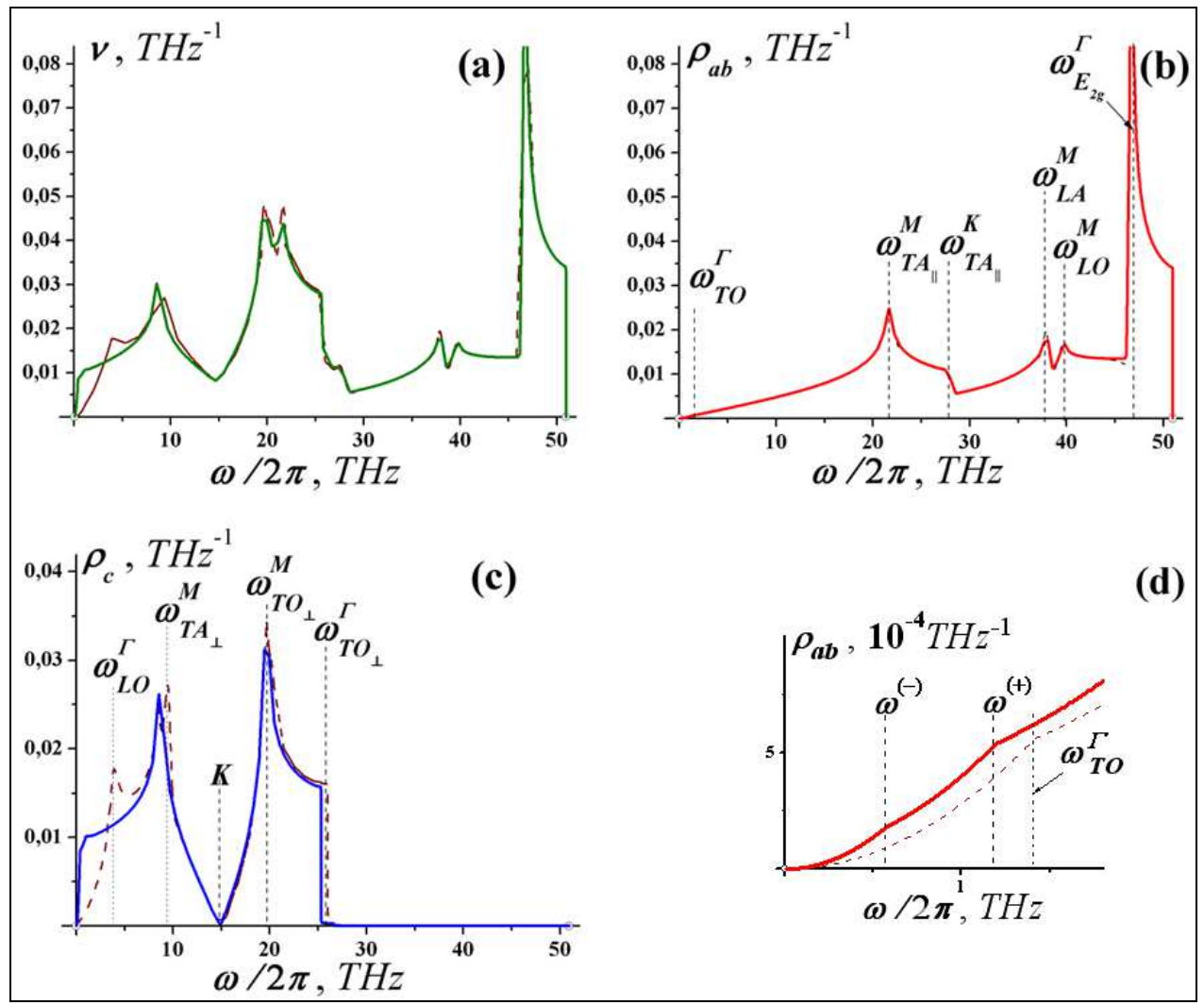

Fig. 7. The phonon density of states of a thin film of graphite, consisting of two graphene monolayers (a) ; the partial contributions to it from the atomic displacement along the basal plane and perpendicular to its direction (b, c, respectively); (d) is low frequency part of (b).

In bigraphene, the transverse phonon modes TA and TO, typical for graphite, degenerate into two discrete levels corresponding to the symmetrical and antisymmetrical displacements of layers. The frequencies of these levels are marked in Fig. $7 \mathrm{~d}$ as $\omega^{(-)}$and $\omega^{(+)}$. The frequency $\omega^{(+)}$in bigraphene corresponds to the same atomic displacements as the frequency $\omega_{T O}(\Gamma)$ on bulk sample, a lower value of $\omega^{(+)}$as compared with $\omega_{T O}(\Gamma)$ is caused by the surface relaxation. The bigraphene spectral density $\rho_{a b}(\omega)$ (Fig. 7d) shows kinks at $\omega=\omega^{(-)}$and $\omega=\omega^{(+)}$. For $\omega>\omega^{(+)}$the spectral density acquires the characteristic two-dimensional appearance $\rho_{a b}(\omega) \sim \omega$.

Starting from very low frequencies, the spectral density $\rho_{c}(\omega)$ acquires the form characteristic of a two-dimensional scalar model, leading to very high values of the meansquare displacements of atoms in the direction perpendicular to layers.

Fig. 8 shows the temperature dependence of the rms amplitudes of atomic displacements of bigraphene (2c), trigraphene (3c for surface layers, $3 c^{\prime}$ for central layer) and bulk sample $(\infty c)$ in the perpendicular direction to the layers, and it also shows that the amplitude of atomic displacements along the graphene layers is almost independent of sample thickness $(a b)$. 
The rms amplitude of atomic displacements along the $c$ axis strongly increases with the decreasing film thickness. At room temperature the amplitude of transverse vibrations of an atom of the trigraphene central layer (curve $3 c^{\prime}$ ) is about twice the value for the bulk sample (curve $\infty \mathrm{c}$ ).

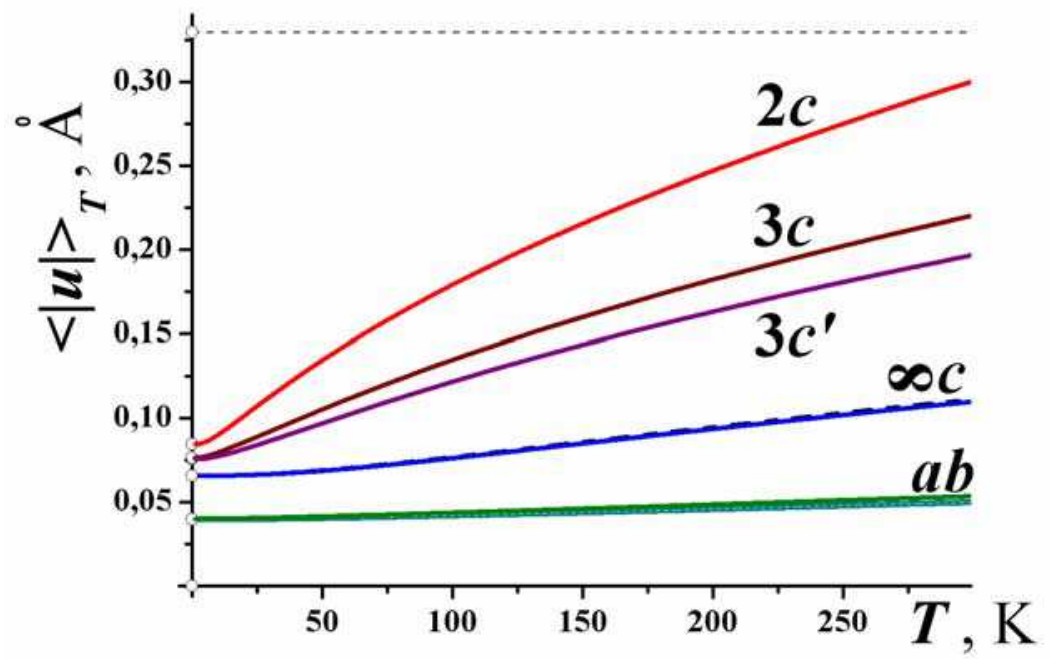

Fig. 8. Temperature dependence of the mean amplitudes of atoms along different crystallographic directions in the films of graphite, consisting of two and three graphene monolayers

The horizontal dashed line in Fig. 8 indicates the mean value of the amplitude of atomic vibrations along the $c$ axis for bulk graphite at $T \approx 3000 \mathrm{~K}$. This temperature is about $1000 \mathrm{~K}$ below the melting temperature of graphite $(T \approx 3800 \pm 50 \mathrm{~K}$ ), therefore at $T \approx 3000 \mathrm{~K}$ the crystal lattice of graphite still has sufficient margin of stability. Bigraphene and trigraphene also have a sufficient margin of stability at room temperature, since the mean square amplitudes of their atomic vibrations are markedly below the dashed line.

\section{Phonon and electron spectra of metal intercalated graphite}

Special interest has been devoted to graphite intercalated by metals, since in such graphitic systems the superconducting transition temperature $T_{\mathcal{c}}$ essentially depends on the type of intercalated metal. For example $T_{c}$ for $C_{6} Y b$ is $6.5 \mathrm{~K}$, while for $\mathrm{C}_{6} \mathrm{Ca}$ it is $11.5 \mathrm{~K}$ (Weller et al., 2005; Emery et al., 2005). It is known that for the formation of the superconducting state the electron-phonon interaction plays crucial role. Since the electronic spectra of these compounds probably do not depend on the type of intercalated metal, $T_{c}$ variations in such compounds are dominantly determined by the peculiarities of their phonon spectra. Therefore it is necessary to analyse in detail the phonon spectra of pure graphite (see section 2 ) and to find out how these spectra vary due to the intercalation with various metals and to determine how these variations depend on the dynamic parameters of both carbon and intercalating metal. 
Both structures mentioned above consist of graphite monolayers between which two dimensional triangle lattices of metals with period $a \sqrt{3}$ are placed. The lattice constant in the direction perpendicular to layers is, in both compounds, $c^{\prime}=4.5 \AA$ (Weller et al., 2005; Emery et al., 2005). The absence of data about acoustical, optical and other properties, which would enable to determine the parameters of interatomic interactions, prompted us to make some assumptions about the values of these interactions. We neglect the carbon-carbon and metal-metal interactions across layers. The interaction of metal atoms within one layer will be considered as a central force, i.e. matrices of force constants have form given in (Feher et al., 2009).

The distance between nearest atoms of metal and carbon is equal to

$$
r_{\mathrm{C}-\mathrm{Me}} \equiv \sqrt{\left(\frac{c^{\prime}}{2}\right)^{2}+\frac{a^{2}}{3}} \approx 2.66 \AA
$$

i.e. it is larger than the distance between second nearest carbon neighbours in graphene monolayer $(a \approx 2.45 \AA)$, but shorter than between third neighbours $(2 a / \sqrt{3} \approx 2.83 \AA$ ). It can be therefore assumed that the potential describing this interaction may be considered as pair and isotropic, i.e. the force constants of interatomic interaction fulfill condition $\beta_{z}\left(r_{\mathrm{C}-\mathrm{Me}}\right)=$ $\beta_{x}\left(r_{\mathrm{C}-\mathrm{Me}}\right)=\beta\left(r_{\mathrm{C}-\mathrm{Me}}\right)$. Since interatomic distances in graphene monolayer do not change due to intercalation, force constants also do not change. Therefore the value of $\beta\left(r_{\mathrm{C}-\mathrm{Me}}\right)$ may be found from the condition of the symmetry of the elastic modulus tensor with respect to a transposition of index pair. This condition has form

$$
\beta\left(\frac{a}{\sqrt{3}}\right)+6 \beta(a)+4 \beta\left(\frac{2 a}{\sqrt{3}}\right)=2\left[\left(\frac{c^{\prime}}{2 a}\right)^{2}-\frac{1}{3}\right] \beta\left(\mathrm{r}_{\mathrm{C}-\mathrm{Me}}\right) .
$$

From this condition we get $\beta\left(r_{\mathrm{C}-\mathrm{Me}}\right) \approx 0.31 \mathrm{~N} / \mathrm{m}$, both for carbon-ytterbium and carboncalcium interactions. Unfortunately, we do not have any data from which force constant $\alpha$ $(r$ C-Me), characterizing central interaction between metal and carbon atoms, may be directly determined. However, on the basis of the values of distances between carbon and metal atoms we estimated that the force constant value lies somewhere in the range from $\alpha\left(r_{\mathrm{C}}\right.$ Me) $\approx 20 \mathrm{~N} / \mathrm{m}$ to $\alpha\left(r_{\mathrm{C}-\mathrm{Me}}\right) \approx 50 \mathrm{~N} / \mathrm{m}$ (Syrkin et al., 2009). We picked up four $\alpha\left(r_{\text {C-Me }}\right)$ values: $20,30,40$, and $50 \mathrm{~N} / \mathrm{m}$.

Fig. 9 shows the frequency dependences of partial contributions to the density of phonon states from displacements of metal and carbon atoms in the direction perpendicular to layers. The areas below dependences corresponding to intercalating metal are hatched. In Fig. 9, the left set show dependences for $\mathrm{C}_{6} \mathrm{Ca}$, the right one for $\mathrm{C}_{6} \mathrm{Yb}$, the force constant $\alpha(r$ C-Me) increases from top to bottom. We see that for $C_{6} C a$ sharp resonance peaks appear on partial contributions from both intercalating metal and carbon. These peaks are shifted, with the increase of $\alpha\left(r_{\mathrm{C}-\mathrm{Me}}\right)$, towards the centre of the frequency range, leading to the increase of density of phonon states near the Brillouin zone's K-point, through which the Fermi level of electrons in graphene passes. For the $C_{6} Y b$ compound ( $Y b$ has more than four times larger atomic mass than $\mathrm{Ca}$ ) the resonance peaks appear at lower frequencies and an apparent 


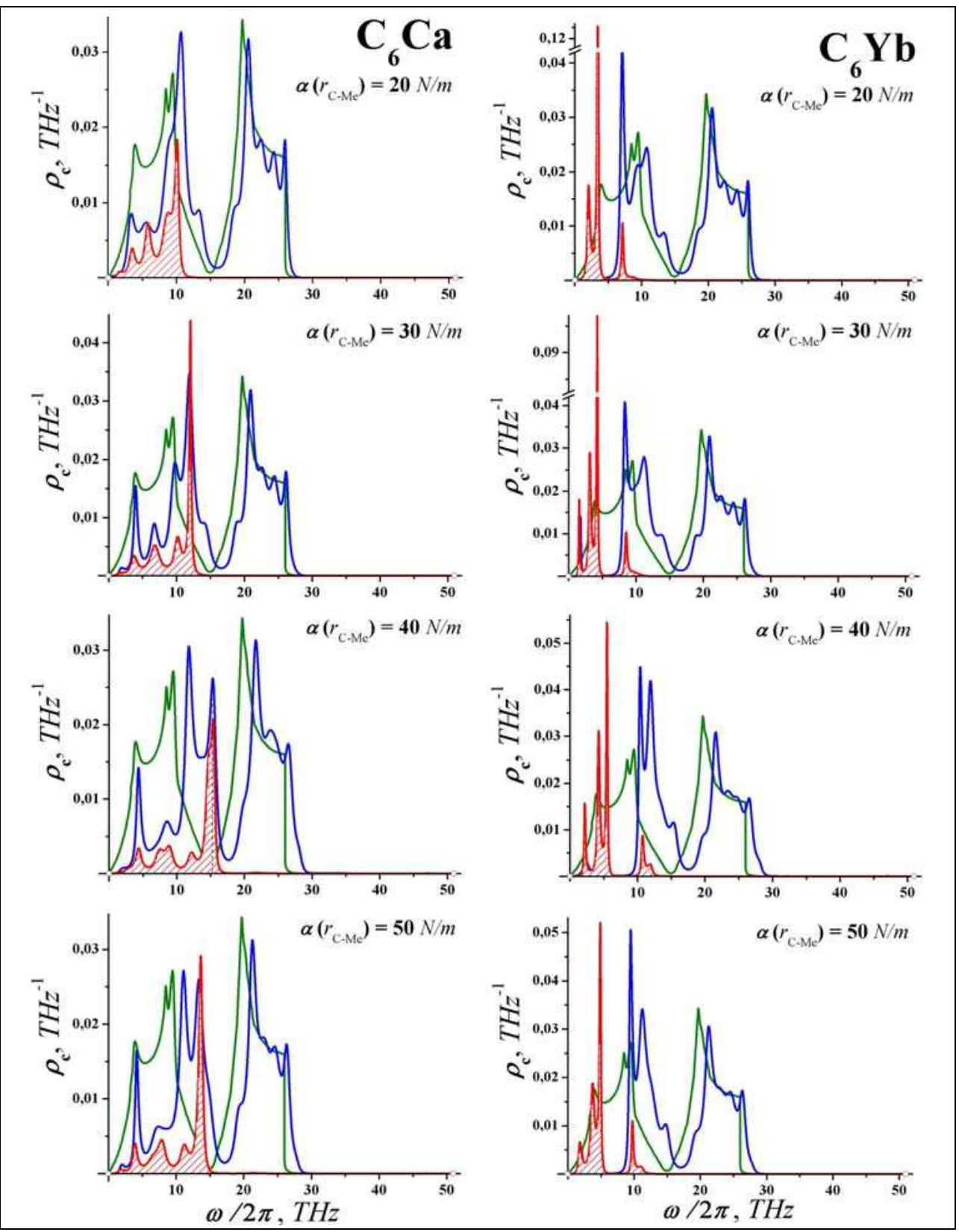

Fig. 9. The partial contributions to the phonon density of states of intercalated graphite (blue lines) from the displacements along the $\mathrm{c}$ axis of the carbon atoms and the metal (red lines with hatched area under the curves). Top to bottom $\alpha\left(r_{\mathrm{C}-\mathrm{Me}}\right)=20,30,40,50 \mathrm{~N} / \mathrm{m}$. Green lines in all the fragments correspond to pure graphite. 
increase of the phonon states density at frequencies near the Brillouin zone's $K$-point is observed only for anomalously large values of $\alpha\left(r_{\mathrm{C}-\mathrm{Me}}\right) \approx 40 \div 50 \mathrm{~N} / \mathrm{m}$ (note that $T_{c}$ for $\mathrm{C}_{6} \mathrm{Ca}$ is almost 1.8 times higher than that for $C_{6} Y b$ ). Note that the sharp resonance peaks in the density of states, which are characteristic for states localized near the isolated impurity atom, appear for metal atoms which form periodic structure. Such a behavior of the spectral densities is due to the weakness of the interaction between remotely spaced atoms. The interatomic distances within the metal layers differ slightly from distances between the atoms of neighbouring layers.

Fig. 10 presents the total phonon density of states for both $\mathrm{C}_{6} \mathrm{Ca}$ (left) and $\mathrm{C}_{6} \mathrm{Yb}$ (right) and partial contributions from intercalating metal (hatched area).

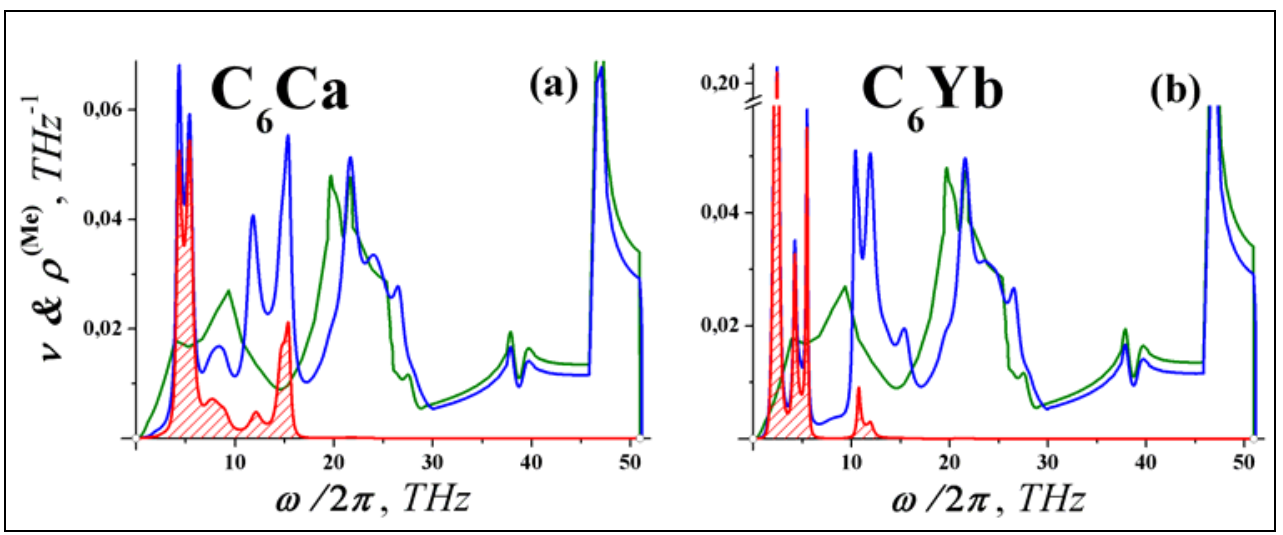

Fig. 10. The phonon density of states of intercalated graphite (blue lines) and the contribution to them of metal atoms (red lines with hatched area under the curves) for $\alpha=50 \mathrm{~N} / \mathrm{m}$. Green lines are the phonon density of states of pure graphite.

Unconventional behavior of phonon subsystem in graphite containing metal intercalated layers may substantially influence its electronic properties and the electron-phonon interaction (EPI), including the superconducting transition temperature. At present time there is much convincing evidence that EPI is principal mechanism of the transition into the superconducting state and this interaction should be taken into account for developing a consistent theory for the description of superconductivity in different types of compounds, including high- $T_{c}$ superconductors (Maksimov, 2008). It follows from the BCS theory (Bardeen et al., 1957) (see also (Maksimov \& Dolgov, 2007)) that the value of $T_{c}$ depends on the basic characteristics of electron and phonon spectra in the following way

$$
T_{c} \propto\left\langle\omega_{p h}\right\rangle \exp \left(-\frac{1}{\lambda}\right)
$$

where $\left\langle\omega_{p h}\right\rangle$ is the mean phonon frequency and $\lambda$ is the EPI constant, which is proportional to the density of electron states on the Fermi surface.

Microscopic analysis of EPI was not performed within this work, but we note that a consistent many-particle theory of EPI already exists, describing both normal and superconducting states of metals (Rainer, 1986; Allen \& Mitrovic, 1982; Maksimov \& 
Dolgov, 2007). However, we can state that to increase $T_{c}$ high phonon frequencies $\left\langle\omega_{p h}\right\rangle$, large values of the constant of the interaction of electrons with these phonons $\lambda$ and high density of electron states on the Fermi surface are needed. Such properties are manifested by, for example, compounds of metals with light elements, such as hydrides (Ashcroft, 2004; Tse et al., 2007), borides (Nagamutsi et al., 2001) carbides and nitrides (Maksimov et al., 2007), since their phonon spectra show high-frequency modes corresponding to the vibrations of light atoms $(\mathrm{H}, \mathrm{B}, \mathrm{C}, \mathrm{N})$. For graphene doped by hydrogen $\mathrm{T}_{\mathrm{c}}$ above the boiling point of liquid nitrogen was recently predicted using the BCS theory (Savini et al., 2010).

Moreover, a detailed analysis has been carried out (see review Maksimov, 2008), showing that electrons in $M g B_{2}$ exhibit very strong interaction with quasi-flexural modes. This is very interesting for describing the evolution of temperature of superconducting transition in the intercalating graphite.

Indeed, the frequency $\left\langle\omega_{p h}\right\rangle$ decreases at intercalating the graphite by metals which have atomic masses essentially larger than carbon. So, the temperature of superconducting transition for graphite intercalated by $L i$ is $1.9 \mathrm{~K}$, which is lower than for graphite intercalated by $\mathrm{Ca}$ or $\mathrm{Yb}$. At the same time the intercalation by $\mathrm{Yb}$ and $\mathrm{Ca}$ increases the mean vibration frequency of quasi-flexural branch. Besides, it is especially interesting that the intercalation by $\mathrm{Ca}$ and $\mathrm{Yb}$ leads to an essential increase of the quasi-flexural phonons number. The quasi-momentum of these phonons corresponds to the $K$ point of the first Brillouin zone, in the vicinity of which the Fermi level is lying in the electron spectra.

The role of defects is very important for the electron-phonon interaction formation, and consequently, for superconducting properties in three dimensional layered crystals. So, in a new type of high temperature layered superconductors ROFeAs (where $R$ is rare-earth element) both the superconductive state and $T_{c}$ are determined by defects (Hosono, 2008). These materials are characterized by layered structure consisting of alternating molecular layers $\mathrm{R}-\mathrm{O}$ and $\mathrm{Fe}$-As. The superconductivity in such compounds originates from electron doping by $\mathrm{F}$ or hole doping by introducing oxygen vacancies (Sadovskii, 2008; Izyumov \&Kurmaev, 2008; Ivanovskii, 2008).

\section{Conclusion}

The results of this chapter allow us to draw following conclusions concerning the electronic spectrum of graphene with defects, the phonon quasi-particle spectra of graphene, bigraphene and metal-intercalated graphite:

i. It was shown that a vacancy in graphene conserves the Dirac singularity of the local density of electronic states in one of the sublattices. Moreover, a quasi-Dirac singularity was also observed in phonon spectra of graphene for atom displacements in the direction perpendicular to layers.

ii. Calculations and description of the phonon spectrum and vibrational characteristics of the bulk graphite and carbon nanofilms (bigraphene and trigraphene) lead to the explanation of the plane shape stability of bigraphene and trigraphene nanofilms at room temperatures.

iii. Analyzing the changes in phonon spectra of graphite intercalated with various metals, using the BCS theory, we have proposed, taking into account dynamic properties of these layered systems, a possible way leading to the increase of $\mathrm{T}_{\mathrm{c}}$. 
Graphite and metallic compounds on its basis seem to be very attractive for complex investigations of electron and phonon spectra, the electron-phonon interaction, and for the study of superconductive transition conditions. The reasons for this attractiveness are, among other:

i. well pronounced flexural modes of graphite, practically coincident (in the same value of quasi-momentum) with the Dirac peculiarities in phonon and electron spectra;

ii. an increased quantity of phonon and electron states near the Fermi level caused by intercalating metals, and

iii. the existence of highly frequency phonon modes, pointing out to their attractiveness.

It should be noted, that in the review (Maksimov, 2008) author argues that in the $M g B_{2}$ compound electrons most strongly interact with flexural modes. This seems to be very interesting for the description of the evolution of the superconducting transition temperature in intercalated graphite, since flexural modes play an essential role in graphite phonon spectra.

Finally, for further analysis of the mechanism of superconducting transition in graphite intercalated with metals more complex investigations of the phonon and electron spectra and of the dependence of the electron-phonon interaction on intercalating metal are needed.

\section{Acknowledgments}

This work was supported by the grants of the Ukrainian Academy of Sciences under the contracts No. 23/07-H, No. 4/10-H and No. 4119 UNTS and by the grants of the Slovak Research and Development Agency under No. APVV-VVCE-0058-07, No. APVV-0006-07 and VEGA No. 1/0159/09.

\section{References}

Allen, P.B. \& Mitrovic, B. (1982). Theory of Superconducting T. In: Solid State Physics, Seitz, F.; Turnbull, D. \& Ehrenreich, H., (Ed.), 1 - 92 Academic, New York

Ashcroff, N.W. (2004). Hydrogen Dominant Metallic Alloys: High Temperature Superconductors? Phys. Rev. Lett., Vol. 92, (2004) 187002 - 6

Bardeen, J.; Cooper, J. L.N. \& Schriffer, J.R. (1957). Theory of Superconductivity. Phys. Rev., Vol. 108, (1957) 1175 - 1204

Belen'kii, G.L.; Belen'kii, E.L.; Salaev, E.Yu. \& Suleimanov, R.A. (1988). Deformation effects in layer crystals. Physics-Uspekhi (Advances in Physical Science) Vol. 31, (1988) 434 - 456

Bena, C. \& Kivelson, S.A. (2005). Quasiparticle scattering and local density of states in graphite. Phys. Rev. B.,Vol. 72, (2005) 125432 - 7

Castro Neto, A.H.; Guinea, F.; Peres, N.M.; Novoselov, K.S. \& Geim, A.K. (2009). The electronic properties of graphene. Rev. Mod. Phys.,Vol 81, (2009) 110 -155

Chen, Li; Zhang, Y. \& Shen, Yui (2007). Self-healing in defective carbon nanotubes: a molecular dynamic study. J. Phys.: Cond. Matter, Vol. 19, (2007) 386212-38616

Dresselhaus, M. S. \& Dresselhaus, G. (2002). Intercalation Compounds of Graphite. Adv. in Phys.,Vol. 51, (2002) 1 - 186

Emery, N.; Herold, C.; d'Astuto, M.; Garcia, V.; Bellina, Ch.; Mareche, J.F.; Lagrange, P. \& Loupias, G. (2005). Superconductivity of Bulk $\mathrm{CaC}_{6}$. Phys. Rev. Lett., Vol. 95, (2005) $087003-7$ 
Ivanovskii, A.L. (2008). New high-temperature superconductors based on rare-earth and transition metal oxyarsenides and related phases. Physics-Uspekhi (Advances in Physical Science), Vol. 51, (2008) 1229 - 1260

Izyumov, Yu.A. \& Kurmaev, E.Z. (2008). FeAs systems: a new class of high-temperature superconductors . Physics-Uspekhi (Advances in Physical Science), Vol. 51, (2008) 1261 $-1286$

Feher, A.; Gospodarev, I. A.; Grishaev, V. I.; Kravchenko, K. V.; Manzheli , E. V. \& Syrkin, E. S. (2009). Effect of defects on the quasiparticle spectra of graphite and graphene. Low Temp. Phys., Vol. 35, (2009) $679-686$

Gospodarev, I. A.; Eremenko V. V.; Kravchenko, K. V.; Sirenko V. A.; Syrkin, E. S. \& Feodosyev, S. B. (2010). Vibrational characteristics of niobium diselenide and graphite nanofilms. Low Temp. Phys., Vol. 36, (2010) 344 - 350

Gospodarev, I. A.; Kravchenko, K. V.; Syrkin, E. S. \& Feodosyev, S. B. (2009). Quasi-twodimensional features in the phonon spectrum of graphite. Low Temp. Phys., Vol. 35, (2009) 589 - 595

Hosono, H. (2008). Layered Iron Pnictide Superconductors: Discovery and Current Status. J. Phys. Soc. Japan, Suppl. C, Vol. 77, (2008) 1 - 8

Kosevich, A.M.; Syrkin, E.S. \& Feodosyev, S.B. (1994). Peculiar Features of Phonon Spectra of Low-Dimensional Crystals. Phys. Low-Dim. Struct., Vol. 3, (1994) 47 - 51

Kossevich, A.M. (1999). The Crystal Lattice (Phonons, Solitons, Dislocations), WILEY-VCH Verlag Berlin $\mathrm{GmBH}$, Berlin

Kotlyar, A.V. \& Feodosyev, S.B. (2006). Local oscillations in crystal lattices with a single connected region of the quasi-continuous phonon spectrum. Low Temp. Phys.,Vol. $32,(2006) 256-262$

Lifshits, I.M. (1945). On the theory of regular pertubations. Reports of AS SSSR, Vol. 48, (1945) $83-86$

Maksimov, E.G. (2008). Room-temperature superconductivity: myth or reality? PhysicsUspekhi (Advances in Physical Science), Vol. 51, (2008) 167 - 170

Maksimov, E.G. \& Dolgov, O.V. (2007). A note on the possible mechanisms of hightemperature superconductivity. Physics-Uspekhi (Advances in Physical Science), Vol. 50, (2007) 933 - 937

Maksimov, E.G.; Ebert S.V.; Magnitskaya M.V. \& Karakozov, A. E. (2007). Ab-initio calculations of the physical properties of transition metal carbides and nitrides and possible routes to high- $\mathrm{T}_{\mathrm{c}}$ superconductivity. J. Exp. Theor. Phys., Vol. 105, (2007) $642-651$

Maultzsch, J.; Reich, S.; Thomsen, C.; Requardt, H. \& Ordejön, P. (2004). Phonon Dispersion in Graphite. Phys. Rev. Lett., Vol. 92, (2004) 075501 - 4

Meyer, J. C.; Geim, A. K.; Katsnelson, M. I.; Novoselov, K. S.; Booth, T. J. \& Roth, S. (2007). The structure of suspended graphene sheets. Nature, Vol. 446, (2007) 60 - 63

Morozov, S.V. \& Firsov, A.A. (2009). Transport properties of bigraphene, Abstract of Reports on the 35th Conference on Low-Temperature Physics, 198. Chernogolovka, Russia, September 29 - October 2, 2009

Nagamutsu, J.; Nagakawa, N.; Muranaka, T.; Zenitani, Y. \& Akimutsu J. (2001). Superconductivity at $39 \mathrm{~K}$ in magnesium diboride. Nature, Vol. 410, (2001) 63 - 65

Nicklow, R.; Wakabayashi, N. \& Smith, H.G. (1972). Lattice Dynamics of Pirolitic Graphite. Phys. Rev. B, Vol. 5, (1972) 4951 - 4962 
Novoselov, K. S.; Geim, A. K.; Morozov S. V.; Diang, D.; Katsnelson, M. I.; Grigorieva, I. V.; Dubonos, S. V. \& Firsov, A. A. (2005). Two-dimensional gas of massless Dirac fermions in graphine. Nature, Vol. 438, (2005) 197 - 201

Peresada, V.I.; Afanasyev, V.N. \& Borovikov, V.S. (1975). On calculation of density of states of single-magnon perturbations in ferromagnetics. Sov. Low Temp. Phys., Vol. 1, (1975) 227 - 232 (and see references here)

Peres, N.M.R; Klironomos, F.D.; Tsai, S.-W.; Lopes dos Santos, J.M.D. \& Castro-Neto, A.H. (2007). Electrom waves in chemistry substituted graphene. Europhys. Lett., Vol. 80, (2007) 67007 - 67012

Rainer, D. (1986). Principles of ab-initio calculations of superconducting transition temperatutes, In: Progress in Low Temperature Physics Vol. X, Brewer, D. F. (Ed.), 371 - 421 Elsevier Science Publisher B.V., Amsterdam

Sadovskii, M.V. (2008). High-temperature superconductivity in iron-based layered compounds. Physics-Uspekhi (Advances in Physical Science), Vol. 51, (2008) 1201 1227

Savini, G.; Ferrari, A.C. \& Giustino, F. (2010). First-Principles Prediction of Doped Graphane as a High-Temperature Electron-Phonon Superconductor. Phys. Rev. Lett., Vol. 105, $037002-4$

Skrypnyk, Yu.V. \& Loktev, V.M. (2006). Impurity effects in a two-dimensional systems with the Dirac spectrum. Phys. Rev. B, Vol. 73, (2006) 241402 - 6

Skrypnyk, Yu.V. \& Loktev, V.M. (2008). Spectral function of graphene with short-range impurity centers. Low Temp. Phys., Vol. 34, (2008) 818 - 825

Shangduan Wu; Lei Jing; Qunxiang Li; Shi Q. W.; Chen, Jie; Xiaoping Wang \& Jinlong Ya (2008). Average Density of States in Disordered Graphene Systems. Phys. Rev. B, Vol. 77, (2008) 195411 - 7

Syrkin, E. S.; Feodosyev, S. B.; Kravchenko, K. V.; Eremenko, A. V.; Kantor, B. Ya. \& Kosevich, Yu. A. (2009). Flexural rigidity of layers and its manifestation in the vibrational characteristics of strongly anisotropic layered crystals. Low Temp. Physics, Vol. 35, (2009) 158 - 162

Tse, J.S.; Yao, Y. \& Tanaka, K. (2007). Novel Superconductivity in Metallic $\mathrm{SnH}_{4}$. Phys. Rev. Lett., Vol. 98, (2007) 117004 - 8

Weller, T.E.; Ellerby, M.; Saxena, S.S.; Smith, R.P. \& Skipper, N.T. (2005). Superconductivity in the intercalated graphite compounds $\mathrm{C}_{6} \mathrm{Yb}$ and $\mathrm{C}_{6}$ Ca. Nat. Phys., Vol. 1, (2005) 39 - 41 


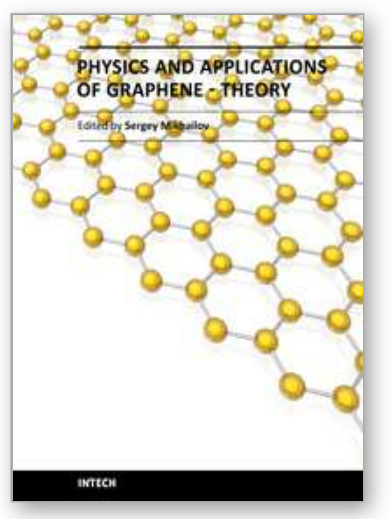

\section{Physics and Applications of Graphene - Theory}

Edited by Dr. Sergey Mikhailov

ISBN 978-953-307-152-7

Hard cover, 534 pages

Publisher InTech

Published online 22, March, 2011

Published in print edition March, 2011

The Stone Age, the Bronze Age, the Iron Age... Every global epoch in the history of the mankind is characterized by materials used in it. In 2004 a new era in material science was opened: the era of graphene or, more generally, of two-dimensional materials. Graphene is the strongest and the most stretchable known material, it has the record thermal conductivity and the very high mobility of charge carriers. It demonstrates many interesting fundamental physical effects and promises a lot of applications, among which are conductive ink, terahertz transistors, ultrafast photodetectors and bendable touch screens. In 2010 Andre Geim and Konstantin Novoselov were awarded the Nobel Prize in Physics "for groundbreaking experiments regarding the two-dimensional material graphene". The two volumes Physics and Applications of Graphene - Experiments and Physics and Applications of Graphene - Theory contain a collection of research articles reporting on different aspects of experimental and theoretical studies of this new material.

\section{How to reference}

In order to correctly reference this scholarly work, feel free to copy and paste the following:

Alexander Feher, Eugen Syrkin, Sergey Feodosyev, Igor Gospodarev and Kirill Kravchenko (2011). QuasiParticle Spectra on Substrate and Embedded Graphene Monolayers, Physics and Applications of Graphene Theory, Dr. Sergey Mikhailov (Ed.), ISBN: 978-953-307-152-7, InTech, Available from:

http://www.intechopen.com/books/physics-and-applications-of-graphene-theory/quasi-particle-spectra-onsubstrate-and-embedded-graphene-monolayers

\section{INTECH}

open science | open minds

\section{InTech Europe}

University Campus STeP Ri

Slavka Krautzeka 83/A

51000 Rijeka, Croatia

Phone: +385 (51) 770447

Fax: +385 (51) 686166

www.intechopen.com

\section{InTech China}

Unit 405, Office Block, Hotel Equatorial Shanghai

No.65, Yan An Road (West), Shanghai, 200040, China

中国上海市延安西路65号上海国际贵都大饭店办公楼 405 单元

Phone: +86-21-62489820

Fax: $+86-21-62489821$ 
(C) 2011 The Author(s). Licensee IntechOpen. This chapter is distributed under the terms of the Creative Commons Attribution-NonCommercialShareAlike-3.0 License, which permits use, distribution and reproduction for non-commercial purposes, provided the original is properly cited and derivative works building on this content are distributed under the same license. 